der - wohl etwas überschätzten - Innovations- und der Integrationswirkung der Referenden ihre Schwächen in der betont minderheitenkritischen Sicht gegenüber „Fremdgruppen“ (zum Beispiel Verbot des Baus von Minaretten 2009). Ein Wort zur Terminologie: Der Rezensent ist nicht glücklich über den - verbreiteten - Begriff der „direkten Demokratie“, weil er suggeriert, eine repräsentative Demokratie sei nur eine indirekte, eine halbherzige.

Das wohl schwächste (nicht: schwache) Kapitel ist das zum Wahlsystem - Vatter unterteilt nach dem formalen Entscheidungs- und nicht nach dem Repräsentationsprinzip, das auf die Effekte zielt. So ordnet er Deutschland als „Mischsystem“ ein, und Länder mit Mehrmannwahlkreisen (wie Griechenland und Spanien), die mehrheitsbildend wirken, zu den Verhältniswahlsystemen. Wie die Abbildung zum Disproportionalitätsgrad (S. 87) verdeutlicht, ist dieser beim „Mischsystem“ in Deutschland erheblich geringer als bei der „Verhältniswahl“ in Griechenland. Dem Rezensenten war die erstaunlich hohe Zahl der Briefwähler (über 80 Prozent!) unbekannt.

Die Schweiz ist „eher freiheitlicher als gleicher, was auch ein Spiegelbild der politischen Kräfteverhältnisse mit einer im internationalen Vergleich starken bürgerlich-liberalen Mehrheit und einer eher schwachen Linken ist" (S. 563, Hervorhebung im Original). In der partiellen Erosion der konkordanzdemokratischen Elemente bei den politischen Eliten sieht der Verfasser wenig Gutes: Transparenz gehe auf Kosten von Konsens. Eine weitere Entfaltung konkurrenzdemokratischer Strukturen sei weder vorteilhaft noch wahrscheinlich. Der Autor betont wohl zu stark die Vorteile der Konkordanzdemokratie.

Vatter hat Linder nicht nur an der Universität Bern abgelöst, sondern auch dessen Werk als „die“ Studie zur Schweiz. Der Feind des Guten ist das Bessere. Die Systematik des Werkes, stärker ausgeprägt als bei Linder, besticht ebenso wie seine Faktentreue und seine politikwissenschaftliche Analysekraft, bezogen auf Methodik und Inhalt. Durch die strikte Orientierung am Kriterienkatalog Lijpharts ist die Studie auch für einen Ländervergleich reizvoll. Die Literaturkenntnis ist stupend, das Urteil eher zurückhaltend, wobei seine Präferenz für ein plebiszitäres Demokratieverständnis auffällt. Spricht der erste Satz des Buches von einer „allgemeinen Einführung in das politische System der Schweiz“ (S. 5), so lautet der letzte der Rezension dagegen: Die Studie vermittelt selbst dem Experten erhellende Erkenntnisse nicht nur zur helvetischen Demokratie im Wandel - „der Vatter“ ist „der neue Linder".

Eckhard Jesse

\title{
Kleine Parlamente: welche Rolle spielt die Größe?
}

Baldwin, Nicholas D.J. (Hrsg.): Legislatures of Small States. A Comparative Study (Series: Library of Legislative Studies), Routledge, London / New York 2013, 216 Seiten, £ 80,-.

Repräsentative Demokratie wird oftmals mit dem Hinweis auf die schiere Größe von Nationalstaaten begründet, die eine Demokratie nach antikem Vorbild verhindere. Ein Blick auf viele Staaten der Welt, die nur wenige Einwohner zählen, beweist allerdings, dass auch diese zu Repräsentationsmechanismen greifen. Der vorliegende, von Nicholas Baldwin herausgegebene Band interessiert sich vornehmlich und ganz allgemein für die Frage, ob die 
geringe Größe von Bedeutung sei (does size matter?). In 15 Beiträgen werden 36 Fälle behandelt, darunter fünf halbsouveräne Einheiten (Bermuda, Gibraltar, Guernsey, Jersey und die Isle of Man) und drei subnationalstaatliche (Hongkong, Nunavut in Kanada und Schottland). Viele Inselstaaten sind vertreten, die allein aufgrund ihrer physischen Gegebenheiten klein sind, so zum Beispiel je zwölf Inseln in der Karibik und im Pazifik. Dazu treten Lesotho, Swaziland, Liechtenstein und Malta. Die meisten der behandelten Fälle haben demokratische Regierungssysteme. Ergänzt werden die Texte von einem Anhang, in dem die wichtigsten Fakten zu den einzelnen Parlamenten zusammengefasst sind.

Es bleibt unklar, welches Kriterium von Kleinheit (zum Beispiel Bevölkerungs- oder Parlamentsgröße) für die Fallauswahl entscheidend war. Dag Anckar verweist in seinem Beitrag einerseits darauf, dass in der Forschung häufiger eine Eine-Million-Grenze genutzt wird oder die Abgrenzung bereits bei 500.000 Einwohnern beginnt, andererseits fasst er nur allgemein auf den Sammelband bezogen zusammen, dass die meisten Texte des Bandes Länder mit weniger als einer halben Million Einwohnern behandeln, aber auch territoriale Einheiten, die viel bevölkerungsreicher sind, Eingang gefunden haben, wie Hongkong mit sieben oder Lesotho mit 1,8 Millionen. Im Gegensatz dazu wird Kleinheit im abschließenden Beitrag von Michael Rush eindeutig auf die Parlamentsgröße bezogen, wenn von „kleinen Parlamenten“ gesprochen wird. Dies leuchtet ein, denn bis auf Lesotho und Schottland haben alle behandelten Legislativen weniger als hundert Abgeordnete. Im Titel des Bandes findet sich hingegen die Beschreibung „Parlamente kleiner Staaten“. Zudem fällt auf, dass die meisten ausgewählten Fälle einen klaren Bezug zu Großbritannien aufweisen - entweder als ehemalige Kolonie wie die karibischen Inseln, als britisches Überseegebiet wie Gibraltar oder als so genannter britischer Kronbesitz wie die Kanalinseln und die Isle of Man. Diese Auswahl kann man treffen; dass sie allerdings nicht weiter reflektiert wird - vor allem in den einleitenden Texten von Baldwin und Anckar und dem Resümee von Rush - befremdet.

Die Qualität der Beiträge variiert, was erstens daran liegt, dass neben Wissenschaftlern auch zahlreiche Praktiker zu Wort kommen, so zwei Vorsitzende von Parlamenten, ein Senator und vier Vertreter aus Parlamentsverwaltungen. Das muss kein Problem sein, führt in diesem Band aber dazu, dass des Öfteren nur Regularien (zum Beispiel zu Malta) oder Fakten referiert werden. Zweitens ist kritisch, dass den Autoren anscheinend kein Leitfaden mit zentralen Fragestellungen oder Gliederungshinweisen vorlag. So konzentrieren sich einige Autoren auf geschichtliche Darstellungen und historische Kontexte, während andere vorwiegend das politische System behandeln. In vielen Texten spielt das Kriterium der Größe überhaupt keine Rolle. Dies ist ein Umstand, der angesichts des Titels der Publikation sehr überrascht. Stattdessen setzen sich etliche Texte mit der Frage auseinander, ob es sich bei den besprochenen Legislativen in der Terminologie der Herausgebers Baldwin um policy-influencing parliaments handelt. Eine löbliche Ausnahme bildet Graham Whites Text über Nunavut der Inuit in Kanada. Er setzt sich ausführlich mit der Kleinheitsproblematik auseinander und verweist zudem auf den ebenfalls wichtigen Kulturfaktor.

Der Kleinheitsfrage nimmt sich dann Rush resümierend an und stellt fest: „But does size of the legislature matter? The short answer is yes, the longer answer more complex." (S. 176) Erstens fällt ihm auf, dass relativ viele der vertretenen Parlamente ohne Fraktionen auskommen, es sich in neun Fällen um Versammlungen ohne Gruppenbildungen handelt (darunter Jersey, Guernsey und die Isle of Man) und dass das Mehrheitswahlsystem nach britischem Muster eingesetzt wird. Zweitens nehmen - berücksichtigend, dass es sich vor- 
nehmlich um parlamentarische Regierungssysteme handelt - die Beziehungen zwischen Exekutive und Legislative in vielen kleinen Parlamenten eigentümliche Züge an, da zahlreiche Abgeordnete Mitglieder der Regierung sind und somit insbesondere die Regierungsfraktionen fast keine Mitglieder unter sich haben, die keine direkte Regierungsverantwortung tragen. Dies hat vermutlich besondere Auswirkungen auf die Mitsteuerung und Kontrolle innerhalb der Regierungsmehrheit, die so nur noch von wenigen Abgeordneten geleistet werden kann. In Gibraltar führt die Aufteilung sogar so weit, dass alle Abgeordneten der Regierungsmehrheit in direkter Regierungsverantwortung stehen. Darüber hinaus ermöglicht die Kleinheit dieser Parlamente eine besondere Nähe und Vertrautheit zwischen allen Beteiligten.

Weitere Auffälligkeiten, die Rush nicht erwähnt, lassen sich nach der Lektüre der Einzelbeiträge zusammentragen:

(1) Es spricht einiges dafür, dass die Repräsentationsbeziehungen der Abgeordneten zu den Wählern von sehr viel Nähe geprägt sind. Viele Parlamentarier repräsentieren nur einbis dreitausend Bürger, so dass gegenseitiges Kennenlernen und von vornherein gegebenes Bekanntsein viel wahrscheinlicher sind als in bevölkerungsreicheren Ländern: So hält Wilfried Marxer für Liechtenstein fest: „,...] Liechtenstein society is closely knit. Political representatives can be easily contacted by any stakeholder and even by individual voters, not only by lobby groups.“ (S. 70) Und Jonathan King konstatiert für die Isle of Man: „With only 2,286 residents per legislator on average, Many residents enjoy a high degree of access to their parliamentary representatives." (S. 123) Inwiefern unterscheiden sich die Repräsentationsbeziehungen zwischen Staaten mit vielen und wenigen Einwohnern? Bieten die Parlamente letzterer andere Kontaktmöglichkeiten an als erstere? Ist man weniger auf mediale Vermittlung angewiesen? Es wäre untersuchenswert, solchen Fragen weiter nachzugehen.

(2) Außerdem arbeiten viele der Parlamente in Teilzeit, so dass ihre Mitglieder nicht nur theoretisch ihren angestammten Berufen neben der Parlamentsarbeit nachgehen können. Für Nunavut wird außerdem festgehalten, dass sich die Berufsstruktur von anderen Parlamenten sehr unterscheidet: So waren zum Beispiel Jäger und LKW-Fahrer vertreten, aber nur ein Abgeordneter besaß einen juristischen Abschluss (allerdings bei einer geringen Gesamtabgeordnetenzahl von 19).

(3) Die hier behandelten „kleinen“ Länder scheinen nicht öfter zu Mitteln direkter Demokratie zu greifen als größere - vielleicht sogar noch weniger (zum Beispiel pazifische Inseln, Anckar S. 41 f.). Das mag angesichts der eingangs formulierten und häufig anzutreffenden Begründung für repräsentative Demokratien überraschen und sollte weiter erforscht werden.

(4) Alle behandelten Parlamente haben Ausschüsse installiert, können aber aufgrund ihrer Kleinheit weniger einsetzen, so dass strukturell die meisten nicht als Ausschussparlamente charakterisiert werden können.

In dieser Zusammenstellung lassen sich Ansatzpunkte für weitere Untersuchungen finden. Zu bedauern ist, dass die Aufgabe, solche Anregungen zu geben, von dem vorliegenden Band nicht erfüllt wurde. Das Buch weist zwar ein interessantes Thema auf - insbesondere wenn man sich vor Augen führt, dass fast die Hälfte aller Parlamente weltweit unter hundert Abgeordnete zählt, sie aber in der Forschung seltener eine Rolle spielt. ${ }^{1}$ Auf das

1 Darauf verweist Rush anhand von Daten der Inter-Parliamentary Union (IPU). Demzufolge haben 44,3 Prozent der Parlamente weltweit weniger als hundert Mitglieder, S. 176. 
eigentliche Thema wird in den einzelnen Texten aber leider häufig gar nicht eingegangen. Es findet sich zusammengewürfeltes Wissen mit durchaus bedenkenswerten Details wie dem Hinweis auf die teilweise funktionale Repräsentation in Hongkongs Erster Kammer, dem Legislativen Rat, oder die ein Mal pro Jahr draußen stattfindenden Sitzungen des Tynwalds auf der Isle of Man, auf der alle im Jahr zuvor verabschiedeten Gesetze offiziell verkündet werden. Es bleibt den Lesern vorbehalten, Wesentliches zusammenzutragen und sinnvoll zusammenzuführen. So kann der Band den selbst gesetzten Anspruch, „problem charting and erecting scaffoldings for future theory-building“ (S. 17), nur bedingt einlösen.

Franziska Carstensen

\section{Auf dem Weg zur europäischen Parteiendemokratie: wie weit ist es noch?}

Poguntke, Thomas, Martin Morlok und Heike Merten (Hrsg.): Auf dem Weg zu einer europäischen Parteiendemokratie (Schriften zum Parteienrecht und zur Parteienforschung, Band 44), Nomos Verlagsgesellschaft, Baden-Baden 2013, 226 Seiten, € 44,-.

Das supranationale Projekt „Europäische Union“ (EU) ist bekanntermaßen nach dem Zweiten Weltkrieg als Antwort auf den Umgang mit Deutschland, den Kalten Krieg und vor allem als Friedensunion initiiert worden. Ein direkt vom „Volk“ gewähltes Parlament gibt es dabei erst seit 1979. Die Weiterentwicklung dieser Institution ist vor allem als PRAktion zu verstehen. Bis zu diesem Zeitpunkt gab es kein stichhaltiges Argument, das den Gedanken eines Elitenprojekts „EU“ hätte widerlegen können. Doch das Parlament und mehr noch seine Akteure, die Parteien, sind „auf europäischer Ebene unabdingbar für die Verwirklichung des Demokratieprinzips“ (S. 7). Die Hauptkritik nach 30 Jahren ist jedoch weiterhin, dass die nationalen Parteien den Ton angeben und dass die Wahlverfahren in den Mitgliedstaaten nicht einheitlich sind. Die Liste ließe sich um viele kleine Kritikpunkte erweitern, offensichtlich ist jedoch, dass von einer gesamteuropäischen Parteiendemokratie keine Rede sein kann. Daher muss gefragt werden, wie weit es noch bis zu dieser ist, beziehungsweise ob es überhaupt vorwärts geht.

Ausgehend von einem Symposium des Instituts für Deutsches und Internationales Parteienrecht und Parteienforschung am 20. und 21. April 2012 an der Heinrich-Heine-Universität Düsseldorf beschäftigt sich der vorliegende Band mit dem Stand und der Entwicklung der europäischen Parteiendemokratie. Die Leitfragen spiegeln dabei das vorhandene Missverhältnis zwischen, in ausreichender Zahl vorhandenen Parteien und nicht als solcher zu erkennender europäischer Parteienpolitik wider: „(1) Ist eine europäische Parteipolitik im Sinne von supranationaler Parteienregierung überhaupt denkbar? Und wenn ja: (2) Welche konstruktiven Veränderungen am europäischen Institutionengefüge und seiner Rechtsordnung sind vorzunehmen, um diese zu ermöglichen?"“ (S. 11).

Antworten hierauf werden zunächst theoretisch, dann rechtlich, im Prozess ihrer Entwicklung und schließlich nach vorn schauend angegangen. Den Anfang machen Thomas Poguntke und Hans H. Klein mit einem Überblick und Überlegungen zu Parteien in der Europäischen Union. 